\title{
Evaluación a los dos meses del alta hospitalaria tras la primera ola de COVID-19: presencia de síntomas persistentes
}

\author{
Assessment of hospitalized covid-19 patients two month after discharge: \\ characterization of persistent symptoms
}

\author{
Blanca Ayuso García, María J Gude González, Antía Pérez López, Yoana Besteiro Balado, Cristina Pedrosa Fraga, \\ Nagore Blanco Cid, Maria M Magariños Losada, Eva Romay Lema \\ Hospital Universitario Lucus Augusti
}

\begin{abstract}
Introduction: a series of symptoms have been reported after COVID-19, which have been encompassed in the so-named "postCOVID syndrome". PostCOVID syndrome is a heterogeneous disorder with an uncertain pathophysiology. The aim of this study is to describe the characteristics and frequence of symptoms after COVID-19 discharge and to analyze the possible implicated factors.
\end{abstract}

Methods: this is an observational propective study with COVID-19 patients hospitalized from March to April 2020. Patients were assessed in an outpatient clinic two months after discharge, and serological, radiological and laboratory workup was conducted. Previous medical history, length of stay (LOS) and intensive care unit (ICU) admission were recorded. Persistent symptons (PS) were defined as those appearing after the acute infection and present at follow-up.

Results: 74 patients were included. Mean age was $66 \pm 13$ years, and $54.4 \%$ patients were men. Six (8.1\%) patients needed ICU admission, and median LOS was 8 (6-12) days. Forty (54.8\%) patients presented PS, the most frequent being fatigue and dyspnea (20.3\% each). $77 \%$ patients presented laboratory abnormalities but just in 11 cases (15.1\%) were they severe. Ten (13.5\%) had radiological abnormalities. 71 (95.9\%) had positive IgG serology. There were no differences between patients with and without PS regarding previous medical history or acute infection course. PS patients had a higher heart rate 83 (75-93) vs 76 65-85) bpm; $\mathrm{p}=0.038$ ) at assessment.

Conclusion: symptoms and laboratory abnormalities are frequent two months after COVID-19, although usually mild. No predictors were found for the presence of PS, but larger studies are needed to ascertain this aseveration. Keywords: COVID-19, persistent COVID, persistent symptoms, follow-up programs.

\section{INTRODUCCIÓN}

Tras el impacto inicial de la pandemia COVID-19, se notificaron después del alta de los pacientes una serie de síntomas heterogéneos, en ocasiones persistentes y en otras de nueva aparición, que se han englobado dentro del conocido como "síndrome post-COVID" o "COVID persistente"1. Estos síntomas se han puesto en relación con las secuelas de la propia infección, con secuelas derivadas de la hospitalización prolongada y la estancia en $\mathrm{UCl}$, con el impacto emocional y psicológico que la pandemia ha supuesto a nivel global y con las patologías previas del paciente que tras una infección severa se pueden hacer más evidentes².

Por todo ello actualmente se están llevando a cabo programas de seguimiento a nivel global de estos pacientes para intentar describir y definir de forma adecuada el "síndrome post-COVID"3. En función de las series, los síntomas persistentes están presentes en un 40$90 \% 4,5$, y conforman un cuadro heterogéneo que comprende desde secuelas físicas hasta funcionales y psicológicas.

\section{RESUMEN}

Introducción: se han notificado tras el alta por COVID-19 una serie de síntomas englobados dentro del llamado "síndrome post-COVID", un cuadro heterogéneo cuya fisiopatología es incierta. Nuestro objetivo es describir las características y frecuencia de síntomas tras el alta y analizar los posibles factores relacionados.

Métodos: estudio observacional prospectivo con pacientes ingresados por COVID-19 durante marzo-abril de 2020. Se evaluó en consulta a los dos meses tras el alta con valoración clínica, analítica, serología y radiografía de tórax. Se recogieron los antecedentes, la estancia hospitalaria y la necesidad de UCl. Se definieron síntomas persistentes (SP) como síntomas que aparecieron desde la infección aguda y que se mantenían al seguimiento. Resultados: se revisaron 74 pacientes. La edad media fue $66 \pm 13$ años, siendo un $51,4 \%$ hombres. Seis $(8,1 \%)$ ingresaron en UCl, y la mediana de estancia fue 8 (6-12) días. Cuarenta (54,8\%) presentaron SP, siendo los más frecuentes astenia y disnea (20,3\% ambos). Un $77 \%$ tenía alteraciones analíticas pero solo en $11(15,1 \%)$ fueron relevantes. Diez (13,5\%) presentaban alteraciones radiológicas y $71(95,9 \%)$ tenían IgG positiva. No hubo diferencias entre los pacientes con y sin SP en sus antecedentes 0 evolución hospitalaria. Los pacientes con SP estaban más taquicárdicos [83 (75-93) Ipm vs 76 (65-85) Ipm; $p=0,038]$, no existiendo diferencias significativas en el resto de variables.

Conclusión: al seguimiento tras la COVID-19 es frecuente la presencia de síntomas 0 alteraciones analíticas, aunque no suelen traducir gravedad. No encontramos variables que predijeran la presencia de los mismos, pero sería interesante analizar cohortes más amplias.

Palabras clave: COVID-19, COVID persistente, síntomas persistentes, programas de seguimiento.

El objetivo de nuestro estudio es describir la situación de nuestros pacientes a los dos meses del alta, determinando las características y frecuencia de síntomas y analizar los posibles factores asociados a su desarrollo.

\section{PACIENTES Y MÉTODOS}

Se ha realizado un estudio de cohortes prospectivo de todos los pacientes ingresados por infección por SARS-CoV-2 confirmado mediante RT-PCR en exudado nasofaríngeo de marzo a abril de 2020 en el Hospital Universitario Lucus Augusti (Lugo). Al alta hospitalaria, se incluyó a todos los pacientes en un buzón administrativo para su posterior citación en consulta de revisión. Se decidió realizar la consulta a los dos meses para asegurar baja probabilidad de infección activa y dar tiempo suficiente a la resolución de la mayoría de síntomas relacionados con la infección aguda. Independientemente de qué servicio o facultativo hubiera atendido al paciente durante el ingreso, se citó a los pacientes en la 
consulta de Enfermedades Infecciosas, siendo todos ellos evaluados por el mismo facultativo. Se excluyeron del estudio los pacientes con deterioro cognitivo grave o incapacidad para desplazarse a consulta, los cubiertos por mutuas aseguradoras privadas y los que rechazaron acudir a la consulta de revisión.

Se ha recogido consentimiento informado para la realización del estudio a todos los pacientes que acudieron a la consulta de revisión. Además de historia clínica y exploración física completa, se realizó analítica (hemograma, bioquímica general, fibrinógeno, dímero D, proteína $\mathrm{C}$ reactiva y ferritina), serología con determinación de títulos de IgG y una radiografía de tórax.

Se revisó la historia clínica electrónica, recogiéndose los antecedentes relevantes, la estancia hospitalaria, así como si tuvieron necesidad de ingreso en la Unidad de Cuidados Intensivos (UCI). Respecto a los valores analíticos se recogieron los correspondientes al día de ingreso, los valores extremos durante el mismo, y los de la consulta de revisión. Se registraron las alteraciones radiológicas si estaban presentes y el título de lgG.

Se definieron síntomas persistentes (SP) como la presencia al seguimiento de síntomas que aparecieron desde la infección aguda y que se mantenían al seguimiento. Se consideró que las variables analíticas estaban alteradas si estaban fuera del rango de valor de normalidad de nuestro laboratorio; así mismo se exploraron variaciones extremas, dos o tres veces por encima o debajo de dicho valor. Las variables cuantitativas se expresan como media con derivación estándar o como mediana con rango intercuartílico; las variables cualitativas se expresan como porcentaje ajustado. Se usó el test de la T de Student o el la U de Mann-Whitney según correspondiera para el análisis de las variables cuantitativas y el test de Chi cuadrado para las cualitativas. Se consideró significación como $p<$ 0,05. El análisis de datos se realizó mediante el paquete estadístico SPSS 25 (IBM, Nueva York, EEUU. 2017).

\section{RESULTADOS}

Durante el periodo estudiado se detectaron 727 resultados positivos en la provincia de Lugo, de los que 125 ingresaron en nuestro centro, falleciendo 17. De los 108 supervivientes, se excluyeron 34 pacientes: 8 pacientes por ser asegurados de sanidad privaday 21 pacientes por deterioro cognitivo o dependencia grave mientras que 5 rechazaron participar en el estudio (figura 1).

Se revisaron finalmente en la consulta 74 pacientes, el 68,5\% de las altas. Sus características basales se describen la tabla 1. La

Figura 1. Diagrama de flujo de los pacientes con COVID-19 evaluados

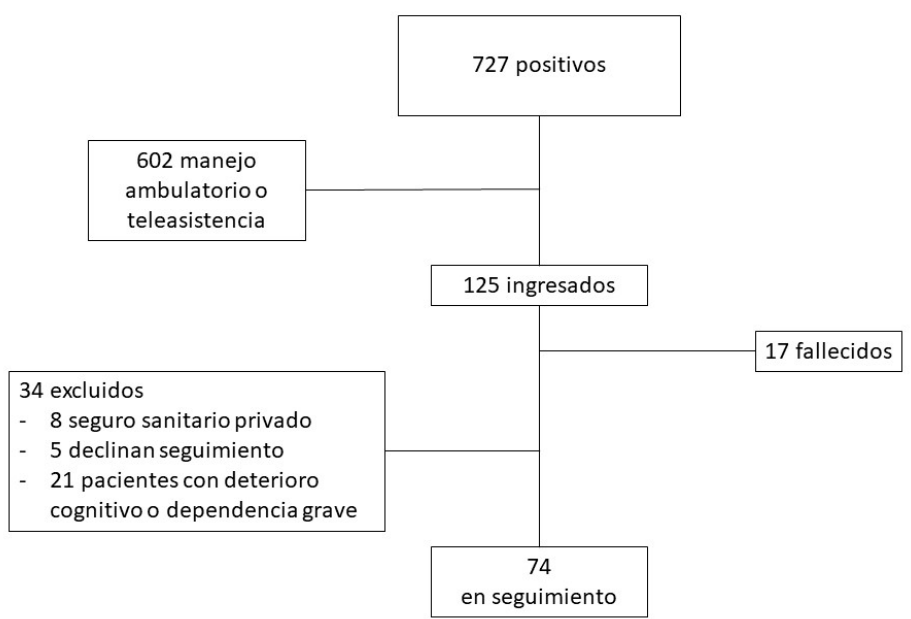

Tabla 1. Características generales de los pacientes

\begin{tabular}{|l|c|c|c|c|}
\hline & $\begin{array}{c}\text { Total } \\
n=74\end{array}$ & $\begin{array}{c}\text { Hombre } \\
n=38\end{array}$ & $\begin{array}{c}\text { Mujer } \\
n=36\end{array}$ & $p$ \\
\hline Características basales \\
\hline Edad (años), media, DE & $66 \pm 13$ & $67 \pm 11$ & $66 \pm 17$ & $>0,1$ \\
\hline Edad > 65 años & $42(56,8 \%)$ & $22(57,9 \%)$ & $20(55,6 \%)$ & $>0,1$ \\
\hline Obesidad (IMC > 30) & $28(41,8 \%)$ & $21(60,0 \%)$ & $7(21,9 \%)$ & 0,002 \\
\hline Antecedentes médicos \\
\hline Hipertensión arterial & $39(52,7 \%)$ & $19(50,0 \%)$ & $20(55,6 \%)$ & $>0,1$ \\
\hline Diabetes mellitus & $22(29,7 \%)$ & $16(42,1 \%)$ & $6(16,7 \%)$ & 0,017 \\
\hline Dislipemia & $35(47,3 \%)$ & $17(44,7 \%)$ & $18(50,0 \%)$ & $>0,1$ \\
\hline $\begin{array}{l}\text { Neumopatía (asma / } \\
\text { EPOC) }\end{array}$ & $8(10,8 \%)$ & $5(13,2 \%)$ & $3(8,3 \%)$ & $>0,1$ \\
\hline Cardiopatía & $9(12,2 \%)$ & $5(13,2 \%)$ & $4(11,1 \%)$ & 1 \\
\hline Inmunosupresión & $4(5,4 \%)$ & $1(2,6 \%)$ & $3(8,3 \%)$ & $>0,1$ \\
\hline Cáncer & $5(6,8 \%)$ & $3(7,9 \%)$ & $2(5,6 \%)$ & 1 \\
\hline Psiquiátrico & $16(21,6 \%)$ & $5(13,2 \%)$ & $11(30,6 \%)$ & 0,067 \\
\hline Evolución durante ingreso & $8(6-12)$ & $9(6-13)$ & $7(5-12)$ & $>0,1$ \\
\hline Estancia media (días) & $30(40,5 \%)$ & $17(44,7 \%)$ & $13(36,1 \%)$ & $>0,1$ \\
\hline Estancia > 10 días & $6(8,1 \%)$ & $4(10,5 \%)$ & $2(5,6 \%)$ & $>0,1$ \\
\hline UCl & $31(41,9 \%)$ & $18(47,4 \%)$ & $13(36,1 \%)$ & $>0,1$ \\
\hline Curso grave* ${ }^{*}$ UCl o estancia > 10 días \\
\hline
\end{tabular}

Tabla 2. Hallazgos en la consulta a los 2 meses del alta

\begin{tabular}{|c|c|}
\hline & $\mathrm{n}=74$ \\
\hline \multicolumn{2}{|l|}{ Síntomas } \\
\hline Astenia & $15(20,3 \%)$ \\
\hline Disnea & $15(20,3 \%)$ \\
\hline Tos & $5(6,8 \%)$ \\
\hline Dolor torácico & $3(4,1 \%)$ \\
\hline Anosmia / ageusia & $1(1,4 \%)$ \\
\hline Mialgias / artralgias & $9(12,2 \%)$ \\
\hline Insomnio & $3(4,1 \%)$ \\
\hline Depresión & $3(4,1 \%)$ \\
\hline Ansiedad & $1(1,4 \%)$ \\
\hline Parestesias & $2(2,7 \%)$ \\
\hline Digestivos & $4(5,4 \%)$ \\
\hline Otros & $8(10,8 \%)$ \\
\hline \multicolumn{2}{|l|}{ Exploración física } \\
\hline Sp02 (\%) & $98(97-98)$ \\
\hline TAS (mmHg) & $150 \pm 23$ \\
\hline TAD $(\mathrm{mmHg})$ & $78 \pm 8$ \\
\hline FC (latidos por minuto, Ipm) & $81 \pm 17$ \\
\hline \multicolumn{2}{|l|}{ Pruebas complementarias } \\
\hline Hemoglobina (g/dL) & $13,8 \pm 1,7$ \\
\hline Leucocitos (cels/mm³) & $6.050 \pm 1.490$ \\
\hline Linfocitos (cels/mm³) & $2.110 \pm 670$ \\
\hline Plaquetas $\left(\mathrm{U} / \mathrm{mm}^{3}\right)$ & $217.300 \pm 57.900$ \\
\hline Fibrinógeno (mg/dL) & $391(346-423)$ \\
\hline Dímero D (ng/mL) & $554(319-824)$ \\
\hline LDH (UI/L) & $177 \pm 33$ \\
\hline Ferritina $(\mathrm{ng} / \mathrm{mL})$ & $86(33-175)$ \\
\hline PCR (mg/L) & $0,70(0,33-3,05)$ \\
\hline Alguna alteración analítica & $57(77 \%)$ \\
\hline Alguna alteración analítica relevante & $11(15,1 \%)$ \\
\hline Alteración en radiografía de tórax & $10(13,5 \%)$ \\
\hline Serología (positiva) & $72(97,3 \%)$ \\
\hline $\lg G(\mathrm{UA} / \mathrm{ml})$ & $193(124-322)$ \\
\hline
\end{tabular}


Tabla 3. Diferencias según presencia o no

de síntomas persistentes en la consulta de revisión

\begin{tabular}{|c|c|c|c|c|}
\hline & $\begin{array}{c}\text { Total } \\
n=74\end{array}$ & $\begin{array}{c}\text { Síntomas } \\
\text { persistentes } \\
n=40\end{array}$ & $\begin{array}{c}\text { Sin síntomas } \\
\text { persistentes } \\
n=34\end{array}$ & $p$ \\
\hline \multicolumn{5}{|l|}{ Exploración física } \\
\hline Sp02 (\%) & $98(97-98)$ & $98(97-98)$ & $98(96-98)$ & 0,05 \\
\hline TAS (mmHg) & $150 \pm 23$ & $144 \pm 25$ & $159 \pm 18$ & $>0,1$ \\
\hline TAD (mmHg) & $78 \pm 8$ & $78 \pm 8$ & $80 \pm 6$ & $>0,1$ \\
\hline FC (lpm) & $80(68-90)$ & $83(75-93)$ & $76(65-85)$ & 0,004 \\
\hline \multicolumn{5}{|l|}{ Radiografía de tórax } \\
\hline Presencia de cualquier alteración & $10(13,5 \%)$ & $5(12,5 \%)$ & $5(14,7 \%)$ & $>0,1$ \\
\hline \multicolumn{5}{|l|}{ Inmunidad } \\
\hline Serología (positiva) & $72(97,3 \%)$ & $40(100,0 \%)$ & $32(94,1 \%)$ & $>0,1$ \\
\hline $\operatorname{lgG}(\mathrm{UA} / \mathrm{ml})$ & $193(124-322)$ & $180(127-327)$ & $200(124-338)$ & $>0,1$ \\
\hline \multicolumn{5}{|l|}{ Alteraciones analíticas } \\
\hline \multicolumn{5}{|l|}{ Anemia } \\
\hline$<13 \mathrm{~g} / \mathrm{dL}^{*}$ & $18(24,3 \%)$ & $11(27,5 \%)$ & $7(20,6 \%)$ & $>0,1$ \\
\hline$<9 \mathrm{~g} / \mathrm{dL}^{* *}$ & $0(0,0 \%)$ & $0(0,0 \%)$ & $0(0,0 \%)$ & - \\
\hline \multicolumn{5}{|l|}{ Linfopenia } \\
\hline$<800 / \mathrm{mm}^{*}$ & $1(1,4 \%)$ & $0(0,0 \%)$ & $1(2,9 \%)$ & \multirow{2}{*}{$>0,1$} \\
\hline$<500 / \mathrm{mm}^{* \star}$ & $0(0 \%)$ & $0(0 \%)$ & $0(0 \%)$ & \\
\hline \multicolumn{5}{|l|}{ Plaquetopenia } \\
\hline$<130.000 / \mathrm{mm}^{*}$ & $4(5,4 \%)$ & $2(5,0 \%)$ & $2(5,9 \%)$ & \multirow{2}{*}{1} \\
\hline$<100.000 / \mathrm{mm}^{\star \star \star}$ & $1(1,4 \%)$ & $1(2,5 \%)$ & $0(0 \%)$ & \\
\hline \multicolumn{5}{|l|}{ Fibrinógeno } \\
\hline$>400 \mathrm{mg} / \mathrm{dL}^{*}$ & $28(38,4 \%)$ & $16(41,0 \%)$ & $12(35,3 \%)$ & $>0,1$ \\
\hline$>600 \mathrm{mg} / \mathrm{dL}$ & $0(0,0 \%)$ & $0(0,0 \%)$ & $0(0,0 \%)$ & - \\
\hline \multicolumn{5}{|l|}{ Dímero D } \\
\hline$>500 \mathrm{ng} / \mathrm{mL}^{*}$ & $40(54,8 \%)$ & $19(48,7 \%)$ & $21(61,8 \%)$ & $>0,1$ \\
\hline$>1000 \mathrm{ng} / \mathrm{mL}^{* *}$ & $8(11,0 \%)$ & $4(10,3 \%)$ & $4(11,8 \%)$ & 1 \\
\hline$>1500$ ng/mL & $4(5,5 \%)$ & $0(0,0 \%)$ & $4(11,8 \%)$ & 0,04 \\
\hline \multicolumn{5}{|l|}{$\mathrm{LDH}$} \\
\hline$>280 \mathrm{UI} / \mathrm{L}^{*}$ & $1(1,4 \%)$ & $1(2,5 \%)$ & $0(0,0 \%)$ & 1 \\
\hline$>562 \mathrm{UI} / \mathrm{L}^{\star *}$ & $1(1,4 \%)$ & $0(0,0 \%)$ & $0(0,0 \%)$ & - \\
\hline \multicolumn{5}{|l|}{ Ferritina } \\
\hline$>322$ ng/mL* & $11(14,9 \%)$ & $6(15,0 \%)$ & $5(14,7 \%)$ & $>0,1$ \\
\hline$>500 \mathrm{ng} / \mathrm{mL}^{\star \star}$ & $3(4,1 \%)$ & $2(5,0 \%)$ & $1(2,9 \%)$ & 1 \\
\hline$>1000$ ng/mL & $2(2,7 \%)$ & $1(2,5 \%)$ & $1(2,9 \%)$ & 1 \\
\hline \multicolumn{5}{|l|}{ PCR } \\
\hline$>5 \mathrm{mg} / \mathrm{L}^{*}$ & $9(12,1 \%)$ & $5(12,5 \%)$ & $4(11,8 \%)$ & 1 \\
\hline$>25 \mathrm{mg} / \mathrm{L}$ & $1(1,4 \%)$ & $0(0 \%)$ & $1(2,9 \%)$ & $>0,1$ \\
\hline Albúmina $<3 \mathrm{~g} / \mathrm{dL}^{*}$ & $1(1,4 \%)$ & $1(2,5 \%)$ & $0(0 \%)$ & 1 \\
\hline \multicolumn{5}{|l|}{ Alteración del perfil hepático } \\
\hline AST>34UI/L // ALT>50/UI/L* & $5(6,8 \%)$ & $4(10,0 \%)$ & $1(2,9 \%)$ & $>0,1$ \\
\hline AST/ALTx2** & $0(0,0 \%)$ & $0(0,0 \%)$ & $0(0,0 \%)$ & - \\
\hline GGT>75UI/L // ALP>138UI/L* & $10(13,5 \%)$ & $5(12,5 \%)$ & $5(14,7 \%)$ & $>0,1$ \\
\hline GGT/ALPX2** & $2(2,7 \%)$ & $1(2,5 \%)$ & $1(2,9 \%)$ & 1 \\
\hline Cualquier alteración analítica & $66(90,4 \%)$ & $37(92,5 \%)$ & $29(87,9 \%)$ & $>0,1$ \\
\hline
\end{tabular}

Sp02: saturación parcial de oxígeno. TAS: tensión arterial sistólica. TAD: tensión arterial diastólica. FC: frecuencia cardiaca. LDH: lactato deshidrogenasa. PCR: proteína

C reactiva. AST: aspartato transaminasa. ALT: alanina transaminasa. GGT: gamma-glutamil transferasa. ALP: fosfatasa alcalina. ( $\left.{ }^{\star}\right)$ Límites de normalidad de laboratorio. 
Figura 2. Evolución de los principales parámetros analíticos (al ingreso, valor extremo durante el mismo, y a los 2 meses del alta) según presencia o no de síntomas persistentes

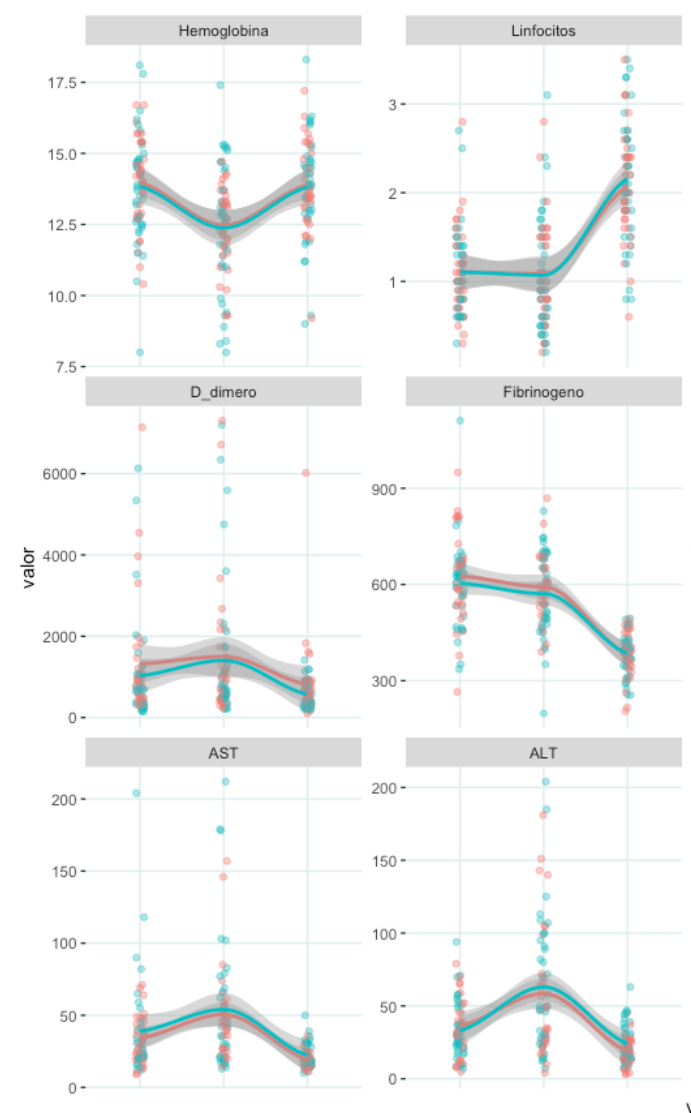

visita
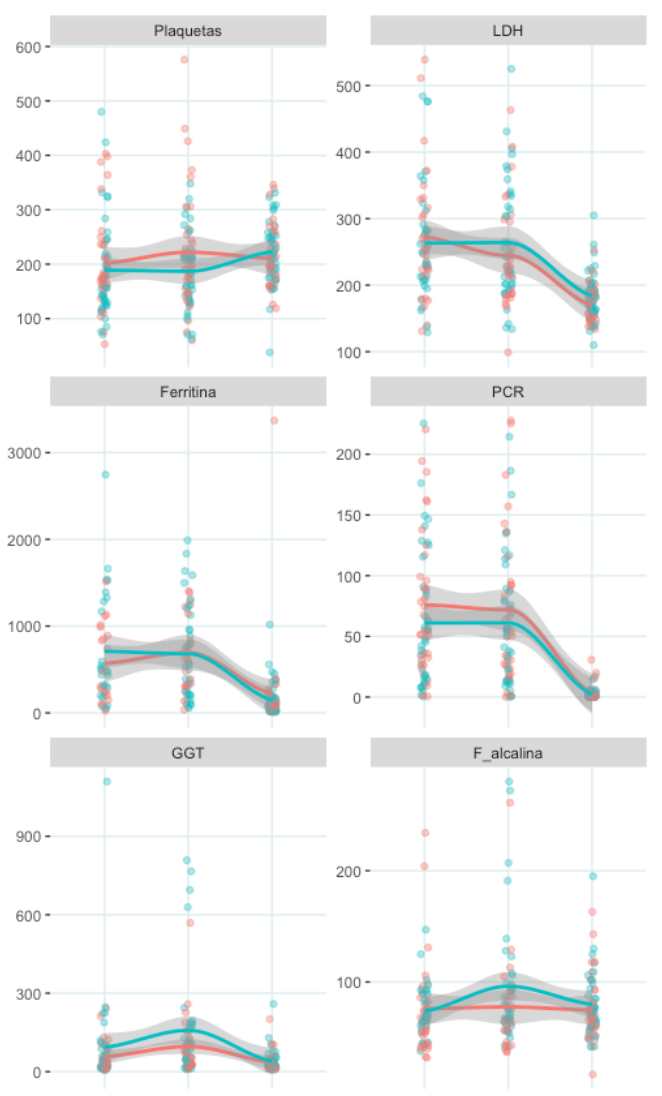

Sintomas persistentes $-\mathrm{Si}-$ No edad media fue de $66.5 \pm 12.8$ años, siendo un $51,4 \%$ hombres. Entre sus antecedentes destacaba que $39(52,7 \%)$ presentaban hipertensión, 22 (29,7\%) diabetes mellitus (DM), y 8 (10,8\%) neumopatía crónica (asma o EPOC). Habían tomado psicofármacos 0 habían tenido diagnóstico previo de patología psiquiátrica 16 pacientes (21,6\%). Durante el episodio de hospitalización 6 pacientes $(8,1 \%)$ precisaron ingreso en UCl para soporte ventilatorio. La mediana de estancia hospitalaria fue de 8 (6-12) días, siendo en un $40,5 \%$ de los casos mayor de 10 días. En el análisis por sexos los hombres presentaban con más frecuencia obesidad ( $60,0 \%$ vs $21,9 \% ; p=0,002)$ y DM ( $42,1 \%$ vs $16,7 \% ; p=0,017)$. Las mujeres tenían más frecuentemente antecedentes psiquiátricos, aunque sin significación estadística (30,6\% vs $13,2 \% ; p=0,067$ ).

En la tabla 2 se resumen los hallazgos al seguimiento. Durante el mismo, 40 pacientes $(54,8 \%)$ tenían algún síntoma persistente desde el alta, siendo los más frecuentes la astenia y la disnea (20,3\% cada uno). Al categorizar los síntomas, 16 pacientes $(21,6 \%)$ presentaban clínica respiratoria (disnea o tos), $6(8,3 \%)$ presentaban clínica psiquiátrica (ansiedad, insomnio o depresión), 10 (13,5\%) síntomas músculo-esqueléticos (mialgias, artralgias, parestesias) y $4(5,4 \%)$ síntomas digestivos; el resto $(51,2 \%)$, presentaban otras categorías (por ejemplo, astenia o anosmia/disgeusia).

De los 16 pacientes con clínica respiratoria, 13 tenían una radiografía normal (81,3\%), siendo en ellos la mediana de Sp02 de 98 (96-98) \%. Un paciente presentaba Saturación de 02 basal de 93\%, pero se objetivó descompensación cardiaca en el momento de la revisión.
Respecto a las alteraciones analíticas, un 77\% tenía alguna alteración en los parámetros analizados, pero solo en 11 pacientes $(15,1 \%)$ estas tenían relevancia (citopenia severa según los criterios HLH-4 ${ }^{6}$, o parámetros inflamatorios y/o transaminasas por encima del doble de la normalidad).

La alteración analítica más frecuente al seguimiento fue la elevación del fibrinógeno (38,4\%) y del dímero D (54,8\%) (Tabla 3). El valor máximo de fibrinógeno fue de $484 \mathrm{mg} / \mathrm{mL}$ (límite mayor de normalidad $400 \mathrm{mg} / \mathrm{dL}$ ). Ocho pacientes (11,0\%) presentaron un dímero D por encima de $1.000 \mathrm{ng} / \mathrm{mL}$; el valor máximo al seguimiento fue $6.000 \mathrm{ng} / \mathrm{mL}$, en un paciente con signos de trombosis venosa profunda en pierna derecha que se confirmó mediante eco-doppler, y el siguiente valor máximo fue de $1.831 \mathrm{ng} / \mathrm{mL}$.

La alteración hematológica más frecuente fue la presencia de anemia (24,3\%; límite inferior de normalidad $13 \mathrm{~g} / \mathrm{dL})$, mientras que sólo un paciente presentó linfopenia (límite de normalidad $800 / \mathrm{mm}^{3}$ )

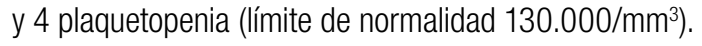

10 pacientes (13,5\%) presentaban alteraciones radiológicas persistentes: dos pacientes con atelectasias laminares, dos pacientes con opacidades periféricas bilaterales, dos pacientes con lesiones fibróticas localizadas y cuatro pacientes con afectación intersticial multilobar. 71 (95,9\%) tenían IgG frente al SARS-CoV-2 positiva.

Reunían criterios de SP 40 pacientes (54,8\%); sus características comparadas con aquellos que no lo presentaron se resumen en la tabla 3. No hubo diferencias relevantes en cuanto a sus antecedentes médicos, estancia hospitalaria o necesidad de ingreso en UCl entre los pacientes con y sin SP. 
Figura 3. Distribución de los títulos de lgG a los 2 meses del alta, según presencia o no de síntomas persistentes. La caja representa mediana con percentil 25-75. El área sombreada representa la distribución de valores de la muestra

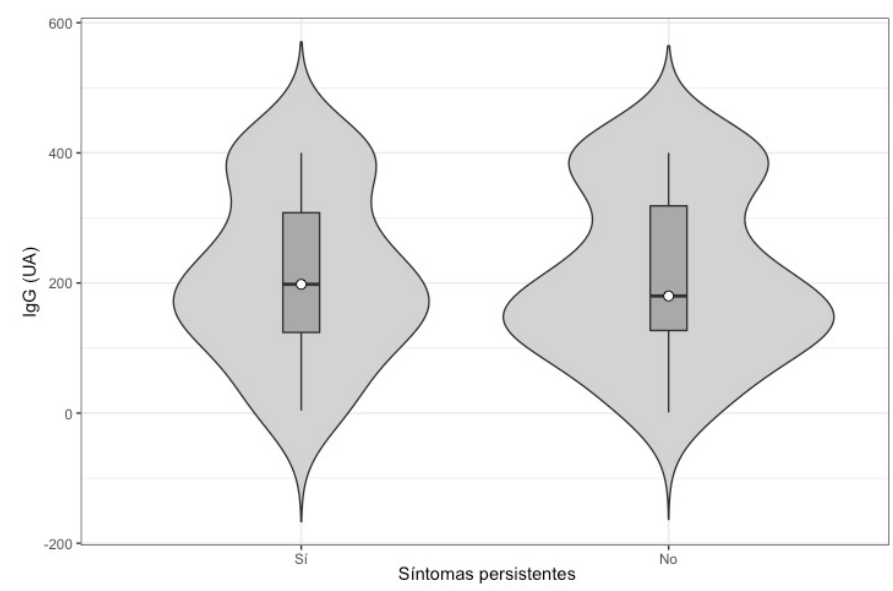

A la valoración en la consulta de seguimiento, los pacientes con SP tenían mayor frecuencia cardíaca [83 (75-93) vs 76 (65-85); $p=0,038]$, no existiendo diferencias significativas en el resto de constantes incluyendo la saturación de oxígeno $(p=0,05)$. No se encontró ninguna diferencia en las alteraciones analíticas entre ambos grupos al seguimiento, y en ambos se observaba una tendencia a la normalización de las mismas tras el alta (figura 2). Tampoco hubo diferencias en cuanto a presencia de alteraciones radiológicas, serología positiva o en los títulos de lgG (figura 3). Se analizaron las alteraciones analíticas al ingreso, así como los valores extremos durante el mismo (anexo 1), y no se encontró ninguna diferencia que pudiese predecir el desarrollo posterior de SP.

\section{DISCUSIÓN}

Nuestro grupo de trabajo ha realizado un seguimiento sistemático a los dos meses de todos los pacientes ingresados por COVID-19 durante la primera ola. Nuestros datos confirman una alta tasa de presencia de síntomas a los dos meses del seguimiento (en torno al $50 \%$ ). Esta tasa es muy variable en la literatura, con datos que van desde el $40 \%$ hasta el 90\% 4,5,7, lo cual está probablemente en relación con la gran heterogeneidad de los estudios existentes, en cuanto a la población de estudio (ambulatoria, hospitalizada, grave; edades, razas y tasa de comorbilidad variables, etc), el diseño del mismo (encuestas telefónicas, encuestas autoadministradas, consultas presenciales) y en cuanto a la forma de abordaje (cribado sólo de síntomas, con analítica o con pruebas radiológicas y de función respiratoria). Cuando se evalúa su evolución en el tiempo, la mayoría tienden a mejorar a los seis meses de seguimiento ${ }^{5}$.

En nuestra muestra, los síntomas más frecuentes fueron la disnea y la astenia, ambos en torno al $20 \%$. Algunos estudios en pacientes exclusivamente hospitalizados han reportado tasas similares a las nuestras ${ }^{8}$ mientras que otras hasta las duplican ${ }^{9}$. Parte de estas diferencias pueden ser debidas al diseño de nuestro estudio, de forma que se han evaluado de forma presencial (no mediante cuestionarios telefónicos o mediante soporte electrónico). En un metanálisis reciente de pacientes tanto ambulatorios como hospitalizados se refleja que en torno un $80 \%$ de los pacientes presentaba uno o más síntomas, de los cuales los más frecuentes eran por orden astenia (58\%), cefalea (44\%), déficit de atención (27\%), alopecia (25\%) y disnea (24\%). Los autores señalan la dificultad de interpretación de los hallazgos dado que la heterogeneidad de los estudios era desde moderada hasta alta ${ }^{10}$.

La alteración analítica al seguimiento más frecuente en nuestro estudio fue la elevación del fibrinógeno y del dímero D. Las alteraciones analíticas de los pacientes con COVID-19 son frecuentes y a veces severas al ingreso, pero suelen mejorar y la mayoría desaparecen con el tiempo. Mandal et al. evaluaron estas alteraciones y no encontraron correspondencia con peor evolución ni con enfermedad subyacente ${ }^{11}$. Aunque existen datos en la literatura de tasa no desdeñables de alteraciones radiológicas y en las pruebas de función respiratoria (principalmente alteración en la capacidad de difusión), estos no siempre traducen síntomas clínicos ${ }^{12,13}$. Además, se ha de tener en cuenta que muchos de estos estudios no disponen de datos previos a la enfermedad por COVID-19 y que series previas de otros coronavirus (SARS y MERS) ya mostraron hallazgos similares, que en su mayoría acababan desapareciendo $0^{14,15}$. Por este motivo, unas guías recientes desaconsejan la realización sistemática de pruebas radiológicas y de función respiratoria a todos los pacientes durante el seguimiento ${ }^{15}$.

Sudre et al. señalaron la edad, el sexo femenino y el número de síntomas al inicio de la fase aguda como posibles predictores de COVID persistente posterior ${ }^{16}$. En nuestra serie, no encontramos diferencias estadísticamente significativas entre los pacientes con SP y los que no lo presentaban en cuanto a sus antecedentes, su evolución durante la hospitalización ni en cuanto a las pruebas complementarias previas. Tampoco nos han sido útiles para discriminar a los pacientes con síntomas persistentes las alteraciones analíticas al seguimiento. El único dato objetivo diferente en ambos grupos fue la discreta mayor frecuencia cardíaca a la valoración en aquellos con SP, sin embargo la alta variabilidad de la frecuencia cardíaca y su influencia por factores externos debe hacer que este dato se evalúe con cautela. Nosotros hemos realizado (aunque no de manera sistemática) ecocardiograma a parte de estos pacientes, no encontrando alteraciones relevantes. Sin embargo un estudio sobre 100 pacientes recuperados tras la infección aguda por el SARS-CoV-2 demostró la presencia de disfunción sistólica y edema e inflamación miocárdica mediante resonancia cardíaca ${ }^{17}$. Habrá que esperar más estudios para valorar qué pruebas de función cardíaca son útiles para la evaluación de pacientes con síntomas persistentes en el seguimiento.

Nuestro estudio tiene fortalezas, como son la evaluación sistemática con las mismas pruebas y por el mismo facultativo a un porcentaje significativo de las altas y el carácter presencial de la misma. Su principal limitación es el pequeño tamaño muestral y que debido a que todos los pacientes corresponden a un mismo centro, los resultados pueden no ser extrapolables. En este sentido, nuestros pacientes se sitúan en el grupo de los de mayor edad respecto a otros, en consonancia con nuestra población. Por otro lado, nuestro estudio no aporta datos del seguimiento a largo plazo, lo que sería interesante para caracterizar mejor el cuadro.

Si bien la atención presencial de estos pacientes permite una adecuada caracterización clínica, sería interesante hacer uso de herramientas estructuradas y validadas para cuantificar las consecuencias a medio plazo de la enfermedad, así como para facilitar la comparación con otras series. Se han propuesto aproximaciones como la $\mathrm{C}_{19-\mathrm{YRS}^{18}}$, y probablemente sean útiles algunas ya establecidas, como la EQ-5D ${ }^{19}$. Su idoneidad ha de ser definida en nuevos estudios.

En conclusión, tras la infección aguda por SARS-CoV-2 pueden persistir o aparecer al seguimiento síntomas variados que en la gran 
mayoría de pacientes no traduce gravedad o enfermedad. También es frecuente la aparición de sintomatología psiquiátrica reactiva al proceso de enfermedad previo. En nuestro estudio, no hemos encontrado variables que permitan predecirlo, aunque sería interesante explorar otras series más amplias en busca de los mismos.

\section{CONSIDERACIONES ÉTICAS}

Se solicitó y obtuvo consentimiento informado a todos los pacientes para la inclusión de sus datos.

\section{FINANCIACIÓN Y CONFLICTO DE INTERESES}

Los autores declaran no haber recibido financiación ni tener conflicto de intereses.

\section{BIBLIOGRAFÍA}

1. COVID-19 rapid guideline: managing the long-term effects of COVID-19. NICE Guidel. 2020; 1-35.

2. Nalbandian A, Sehgal K, Gupta A, Madhavan M V, McGroder C, Stevens JS, et al. Post-acute COVID-19 syndrome. Nat Med. 2021;27(4):601-615.

3. Landi F, Gremese E, Bernabei R, Fantoni M, Gasbarrini A, Settanni CR, et al. PostCOVID-19 global health strategies: the need for an interdisciplinary approach. Aging Clin Exp Res. 2020;32(8):1613-20.

4. Carfi A, Bernabei R, Landi F. Persistent Symptoms in Patients After Acute COVID-19. JAMA. 2020;324(6):603-5.

5. Wang $X, X u H$, Jiang $H$, Wang L, Lu C, Wei X, et al. Clinical features and outcomes of discharged coronavirus disease 2019 patients: A prospective cohort study. Qjm. 2020;113(9):657-65

6. Henter J, Horne A, Arico M, Egeler R, Filipovich A, Imashuku S, et al. HLH-2004 Diagnostic and Therapeutic Guidelines for Hemophagocytic Lymphohistiocytosis. PediatrBloodCancer. 2008;48(2):124-31.

7. Moreno-pérez 0 , Merino E, Leon-ramirez J, Andres M, Manuel J, Arenas-jiménez J, et al. Post-acute COVID-19 syndrome. Incidence and risk factors: A Mediterranean cohort study. J Infect. 2021;82(3):378-83.

8. Prescott H. Outcomes for Patients Following Hospitalization for COVID-19. JAMA.2021;325(15):1511-1512.

9. Halpin SJ, Mclvor C, Whyatt G, Adams A, Harvey 0, McLean L, et al. Postdischarge symptoms and rehabilitation needs in survivors of COVID-19 infection: A crosssectional evaluation. J Med Virol. 2021;93(2):1013-22.

10. López-León S, Wegman-Ostrosky T, Perelman C, Sepulveda R, Rebolledo PA, Cuapio A, et al. More than 50 Long-Term Effects of COVID-19: A Systematic Review and Meta-Analysis. SSRN Electron J. 2021;1-22.

11. Mandal S, Barnett J, Brill SE, Brown JS, Denneny EK, Hare SS, et al. "LongCOVID": A cross-sectional study of persisting symptoms, biomarker and imaging abnormalities following hospitalization for COVID-19. Thorax. 2020;1-3.

12. Huang C, Huang L, Wang Y, Li X, Ren L, Gu X, et al. 6-month consequences of COVID-19 in patients discharged from hospital: a cohort study. Lancet. 2021;397(10270):220-32.

13. Zhao Y miao, Shang Y min, Song W bin, Li Q quan, Xie H, Xu Q fu, et al. Followup study of the pulmonary function and related physiological characteristics of COVID-19 survivors three months after recovery. EClinicalMedicine. 2020; 25:100463.

14. Ahmed H, Patel K, Greenwood DC, Halpin S, Lewthwaite P, Salawu A, et al. Long-term clinical outcomes in survivors of severe acute respiratory syndrome (SARS) and Middle East respiratory syndrome (MERS) coronavirus outbreaks after hospitalisation or ICU admission: A systematic review and meta-analysis. J Rehabil Med. 2020;52(5).

15. George PM, Barratt SL, Condliffe R, Desai SR, Devaraj A, Forrest I, et al. Respiratory follow-up of patients with COVID-19 pneumonia. Thorax. 2020;75(11):1009-16.

16. Sudre C, Murray B, Varsavsky T, Graham M, Penfold R, Bowyer R, et al. Attributes and predictors of long COVID. Nat Med. 2021;27(4):626-631.

17. Puntmann VO, Carerj ML, Wieters I, Fahim M, Arendt C, Hoffmann J, et al. Outcomes of Cardiovascular Magnetic Resonance Imaging in Patients Recently Recovered from Coronavirus Disease 2019 (COVID-19). JAMA Cardiol. 2020;5(11):1265-73

18. Sivan M, Halpin S, Hollingworth L, Snook N, Hickman K, Clifton IJ. Development of an integrated rehabilitation pathway for individuals recovering from COVID-19 in the community. J Rehabil Med. 2020;52(8)

19. Garrigues $E$, Janvier P, Kherabi Y, Hamon A, Gouze H, Doucet L, et al. Postdischarge persistent symptoms and health-related quality of life after hospitalization for COVID-19. J Infect. 2020;81(6):e4-6.
Anexo 1. Parámetros analíticos, valores extremos durante el episodio de hospitalización

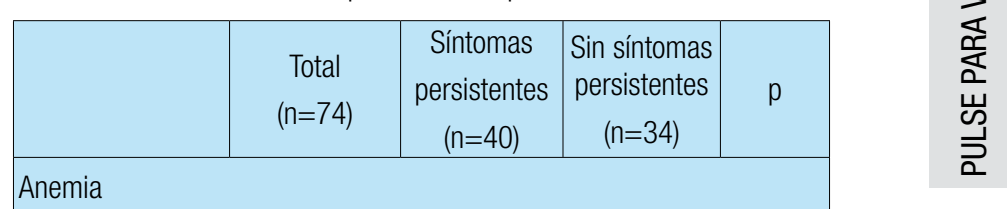

\begin{tabular}{|c|c|c|c|c|}
\hline \multicolumn{5}{|l|}{ Anemia } \\
\hline$<13 \mathrm{~g} / \mathrm{dL}$ & $43(58,1 \%)$ & $25(62,5 \%)$ & $18(52,9 \%)$ & $>0,1$ \\
\hline$<9 g / d L$ & $4(5,4 \%)$ & $4(10,0 \%)$ & $0(0,0 \%)$ & $>0,1$ \\
\hline \multicolumn{5}{|l|}{ Linfopenia } \\
\hline$<800 / \mathrm{mm} 3$ & $25(33,3 \%)$ & $15(37,5 \%)$ & $10(29,4 \%)$ & $>0,1$ \\
\hline$<500 / \mathrm{mm} 3$ & $10(13,5 \%)$ & $6(15,0 \%)$ & $4(11,8 \%)$ & $>0,1$ \\
\hline \multicolumn{5}{|l|}{ Plaquetopenia } \\
\hline$<130.000 / \mathrm{mm} 3$ & $12(16,2 \%)$ & $7(17,5 \%)$ & $5(14,7 \%)$ & $>0,1$ \\
\hline$<100.000 / \mathrm{mm} 3$ & $7(9,5 \%)$ & $4(10,0 \%)$ & $3(8,8 \%)$ & 1 \\
\hline \multicolumn{5}{|c|}{ Elevación fibrinógeno } \\
\hline$>400 \mathrm{mg} / \mathrm{dL}$ & $64(94,1 \%)$ & $34(91,9 \%)$ & $30(96,8 \%)$ & $>0,1$ \\
\hline$>600 \mathrm{mg} / \mathrm{dL}$ & $31(45,6 \%)$ & $17(45,9 \%)$ & $14(45,2 \%)$ & 1 \\
\hline \multicolumn{5}{|l|}{ Elevación dímero $\mathrm{D}$} \\
\hline$>500 \mathrm{ng} / \mathrm{mL}$ & $54(75,0 \%)$ & $28(71,8 \%)$ & $26(78,8 \%)$ & $>0,1$ \\
\hline$>1000 \mathrm{ng} / \mathrm{mL}$ & $32(44,4 \%)$ & $15(38,5 \%)$ & $17(51,5 \%)$ & $>0,1$ \\
\hline$>1500 \mathrm{ng} / \mathrm{mL}$ & $19(26,4 \%)$ & $10(25,6 \%)$ & $9(27,3 \%)$ & $>0,1$ \\
\hline \multicolumn{5}{|l|}{ Elevación LDH } \\
\hline$>280 \mathrm{UI} / \mathrm{L}^{*}$ & $23(33,8 \%)$ & $14(36,8 \%)$ & $9(30,0 \%)$ & $>0,1$ \\
\hline$>562$ UI/L & $1(1,4 \%)$ & $0(0,0 \%)$ & $1(3,3 \%)$ & $>0,1$ \\
\hline \multicolumn{5}{|l|}{ Elevación ferritina } \\
\hline$>322 \mathrm{ng} / \mathrm{mL}^{*}$ & $39(67,2 \%)$ & $21(61,8 \%)$ & $18(75,0 \%)$ & $>0,1$ \\
\hline$>500 \mathrm{ng} / \mathrm{mL}$ & $32(55,2 \%)$ & $17(50,0 \%)$ & $15(62,5 \%)$ & $>0,1$ \\
\hline$>1000 \mathrm{ng} / \mathrm{mL}$ & $16(27,6 \%)$ & $9(26,5 \%)$ & $7(29,2 \%)$ & $>0,1$ \\
\hline \multicolumn{5}{|l|}{ Elevación PCR } \\
\hline$>5 \mathrm{mg} / \mathrm{L}^{*}$ & $67(91,8 \%)$ & $37(92,5 \%)$ & $30(90,9 \%)$ & $>0,1$ \\
\hline$>50 \mathrm{mg} / \mathrm{L}$ & $40(54,8 \%)$ & $21(52,5 \%)$ & $19(57,6 \%)$ & $>0,1$ \\
\hline Albúmina $<3 \mathrm{~g} / \mathrm{dL}$ & $9(14,5 \%)$ & $7(19,4 \%)$ & $2(7,7 \%)$ & $>0,1$ \\
\hline \multicolumn{5}{|c|}{ Alteración del perfil hepático } \\
\hline $\begin{array}{l}\text { AST>34UI/L // } \\
\text { ALT>50/UI/L }\end{array}$ & $44(66,7 \%)$ & $24(63,2 \%)$ & $20(71,4 \%)$ & $>0,1$ \\
\hline AST/ALTx2** & $19(29,7 \%)$ & $12(32,4 \%)$ & $7(25,9 \%)$ & $>0,1$ \\
\hline $\begin{array}{l}\text { GGT>75UI/L // } \\
\text { ALP>138UI/L }\end{array}$ & $32(50,8 \%)$ & $21(58,3 \%)$ & $11(40,7 \%)$ & $>0,1$ \\
\hline GGT/ALPX2 ${ }^{* *}$ & $18(29,0 \%)$ & $12(33,3 \%)$ & $6(23,1 \%)$ & $>0,1$ \\
\hline
\end{tabular}

